\title{
PATHS - Exploring Digital Cultural Heritage Spaces
}

\author{
Mark Hall ${ }^{2}$, Eneko Agirre ${ }^{1}$, Nikolaos Aletras ${ }^{2}$, Runar Bergheim ${ }^{3}$, Konstantinos \\ Chandrinos $^{4}$, Paul Clough ${ }^{2}$, Samuel Fernando ${ }^{2}$, Kate Fernie ${ }^{5}$, Paula Goodale ${ }^{2}$, \\ Jillian Griffiths ${ }^{5}$, Oier Lopez de Lacalle ${ }^{6}$, Andrea de Polo ${ }^{7}$, Aitor Soroa ${ }^{1}$, Mark \\ Stevenson ${ }^{2}$ \\ 1 e.agirrela.soroa@ehu.es \\ University of the Basque Country, Donostia, Spain \\ 2 naletras1|p.d.clough|s.fernando|p.goodale|m.mhall|m.stevenson@sheffield.ac.uk \\ Sheffield University, Sheffield, United Kingdom \\ 3 runarbe@gmail.com \\ Asplan Viak Internet AS, Norway \\ 4 chandrinos@gmail.com \\ iSieve Technologies, Greece \\ 5 kfernie27@gmail.com,jillian.griffiths@mdrprojects.com \\ MDR Partners, United Kingdom \\ 6 oier.lopezdelacalle@gmail.es \\ Basque Foundation for Science / University of Edinburgh, Bilbao, Spain / Edinburgh, \\ United Kingdom \\ 7 andrea@alinari.it \\ Alinari, Florence, Italy
}

\begin{abstract}
Large amounts of digital cultural heritage $(\mathrm{CH})$ information have become available over the past years, requiring more powerful exploration systems than just a search box. The PATHS system aims to provide an environment in which users can successfully explore a large, unknown collection through two modalities: following existing paths to learn about what is available and then freely exploring.
\end{abstract}

\section{Introduction}

Large amounts of digital cultural heritage $(\mathrm{CH})$ information have become available over the past years, especially with the rise of aggregators such as Europeana $^{8}$, the European aggregator for museums, archives, libraries, and galleries. These large collections present a challenge to the new user, primarily in discovering what items are present in the collection. In current systems support for item discovery is mainly through the standard search paradigm [10], which is well suited for $\mathrm{CH}$ professionals who are highly familiar with the collections, subject areas, and have specific search goals. However, for new users who do not have a good understanding of what is in the collections, what search keywords to use, and have vague search goals, this method of access is unsatisfactory.

\footnotetext{
8 http://www.europeana.eu
} 
Alternative item discovery methodologies are required to introduce new users to digital $\mathrm{CH}$ collections [5, 9]. Walden's Paths [8] pioneered the idea of providing users with manually curated paths through the collection. Similarly [7] describe an automatic route-suggestion system for web-pages with the same aim of guiding novice users. To avoid relying on user input to create the initial paths a variety of visualisation techniques [3] have been devised to provide the user with an overview over the collection [6]. Such visualisations provide improved access [11] as long as they ensure that similar items are grouped together [4]. Another benefit is that they promote serendipitous discovery of items [1].

\section{The PATHS System}

The PATHS system presented in this demonstration aims to provide a collection discovery environment that combines the best aspects of these approaches into an integrated, seamless system. The system has two main modes: first, it allows the user to follow existing paths through the collection, a more focused variant of Walden's Paths [8]; second, it allows the user to easily branch off a path and explore the collection on their own. The idea is to assist the novice user in transitioning from consuming existing paths, to exploring on their own, and finally creating paths for others to consume.

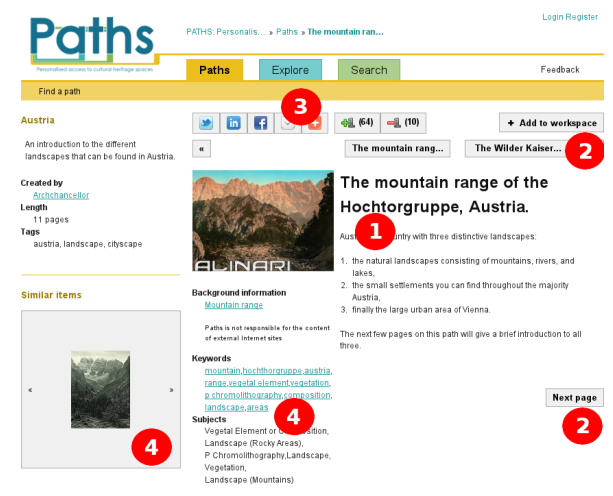

Fig. 1. The path-following interface consists of the item itself with its narrative (1), path navigation (2), social features (3), and exploration starting points (4).

Figure 1 shows the user following an existing path. Such a path consists of a linear sequence of items taken from the collection, where each item consists of its original meta-data plus a title and description (1) which the path author uses to create a narrative that the user can navigate along (2). In order to entice the user away from the path and transition them into the exploration phase, similar items and links to the item's topics and keywords are provided (4).

To support the exploration phase the system provides a number of different exploration modalities (Fig. 2), enabling the user to choose the exploration 
methodology that best suits their personal style and their current task or goal. Currently the system supports exploration via standard vocabularies (1), visual representations of hierarchical topic structures (2), and keyword clouds (3). Additionally, as in the path following interface, the provision of similar items provides a horizontal exploration network.

As the user explores the collection they can save the items, vocabulary entries, topics, or keywords to their workspace. The user can then form these into a path to share with their friends, colleagues, or everybody. Sharing and social interaction have become core ideas in the interaction with cultural heritage [2] and the PATHS system follows this trend by allowing the users to share, tag, and comment on anything they find while exploring the collection (Fig. 1 (3)).

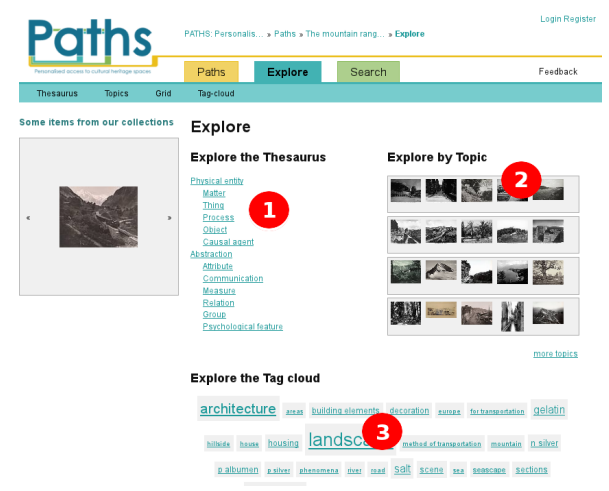

Fig. 2. Exploration interface with the three primary exploration methodologies: standard vocabularies (1), visual topics (2), keyword clouds (3).

The PATHS system is built using a three-tier architecture consisting of a set of four component web-services that access a shared data storage and are tied together by the front-end user-interfaces (Fig. 3). The components provide acces to user-profiles, individual items, exploration visualisation, and path following and creating functionality that can be shared across a number of user-interfaces. The demo will focus on the web-based user-interface, but mobile apps and javascript components to enable integration into other sites are also under development.

\begin{tabular}{|c|c|c|c|}
\hline \multicolumn{4}{|c|}{ Shared Data Storage } \\
\hline $\begin{array}{l}\text { User } \\
\text { profile }\end{array}$ & $\begin{array}{l}\text { Item } \\
\text { access }\end{array}$ & $\begin{array}{c}\text { Exploration } \\
\text { visualisation }\end{array}$ & $\begin{array}{l}\text { Path creation } \\
\text { and following }\end{array}$ \\
\hline Web- & d Demo & Mobile Apps & JS Components \\
\hline
\end{tabular}

Fig. 3. The three-tier architecture used by the PATHS system 


\section{Demo Description}

The PATHS demo will provide a guided tour to the PATHS system and demonstrate how a user can explore the collection, collect items and then form these into a path for others to follow. Participants can then explore the prototype system on their own, which will be provided at http://www.paths-project.eu. The demo will provide access to a sub-set of the data available in Europeana, covering approximately 1.7 million items from the English and Spanish collections.

\section{Acknowledgements}

The research leading to these results has received funding from the European Community's Seventh Framework Programme (FP7/2007-2013) under grant agreement $\mathrm{n}^{\circ} 270082$. We acknowledge the contribution of all project partners involved in PATHS (see: http://www.paths-project.eu).

\section{References}

1. Beale, R. Supporting serendipity: Using ambient intelligence to augment user exploration for data mining and web browsing. International Journal of HumanComputer Studies 65, 5 (2007), 421 - 433. Ambient intelligence: From interaction to insight.

2. Bernstein, S. Where do we go from here? continuing with web 2.0 at the brooklyn museum. In Museums and the Web 2008: the international conference for culture and heritage on-line (2008).

3. Butavicius, M. A., And Lee, M. D. An empirical evaluation of four data visualization techniques for displaying short news text similarities. International Journal of Human-Computer Studies 65, 11 (2007), 931 - 944.

4. Chen, C., Cribin, T., Kuljis, J., And Macredie, R. Footprints of information foragers: behaviour semantics of visual exploration. International Journal of Human-Computer Studies 57, 2 (2002), 139 - 163.

5. Geser, G. Resource discovery - position paper: Putting the users first. Resource Discovery Technologies for the Heritage Sector 6 (2004), 7-12.

6. HornbeK, K., And Hertzum, M. The notion of overview in information visualization. International Journal of Human-Computer Studies 69, 7-8 (2011), 509 525.

7. Johchims, T., Freitag, D., Mitchell, T., Et Al. Webwatcher: A tour guide for the world wide web. In International Joint Conference on Artificial Intelligence (1997), vol. 15, Lawrence Erlbaum Associates Ltd, pp. 770-777.

8. Shipman III, F., Furuta, R., Brenner, D., Chung, C., And Hsieh, H. Using paths in the classroom: experiences and adaptations. In Proceedings of the ninth ACM conference on Hypertext and hypermedia (1998), ACM, pp. 267-270.

9. Steemson, M. Digicult experts seek out discovery technologies for cultural heritage. Resource Discovery Technologies for the Heritage Sector 6 (2004), 14-20.

10. Sutcliffe, A., And EnNis, M. Towards a cognitive theory of information retrieval. Interacting with Computers 10 (1998), 321-351.

11. Westerman, S. J., And Cribbin, T. Mapping semantic information in virtual space: dimensions, variance and individual differences. International Journal of Human-Computer Studies 53, 5 (2000), 765 - 787. 